\title{
Sleep-Related Violence, Self-Mutilation, and Dissociative Experiences
}

\author{
Mehmet Yucel Agargun ${ }^{1 *}$, Lutfullah Besiroglu², Mustafa Gulec ${ }^{2}$, Adem Aydin $^{3}$, Yavuz Selvi ${ }^{4}$ \\ ${ }^{1}$ Istanbul Medipol University, School of Medicine, Department of Psychiatry, Istanbul, Turkey \\ ${ }^{2}$ Katip Celebi University, School of Medicine, Department of Psychiatry, Izmir, Turkey \\ ${ }^{3}$ Meram University, School of Medicine, Department of Psychiatry, Konya, Turkey \\ ${ }^{4}$ Selcuk University, School of Medicine, Department of Psychiatry, Konya, Turkey
}

\begin{abstract}
Twenty-eight subjects with and 46 subjects without sleep-related violence (VBS) were evaluated regarding the presence of self-mutilative behaviors (SMBs). The subjects with and without VBS were compared using dissociative experiences scale (DES). The subjects with VBS had higher mean DES score and more frequent SMBs than those without VBS. These findings suggest that dissociation may be related to both daytime SMBs and violent behaviors during sleep in young population.
\end{abstract}

Keywords: dissociation, sleep-related violence, self-mutilation, dissociative experiences

\section{INTRODUCTION}

The violent behavior during sleep (VBS) involves a broad range of behaviors: self-mutilative behaviors ( $S M B s$ ), sexual assault, murder attempt, murder and suicide, and can be directed to other subjects, to objects, or to self (Ohayon, 2000). Recently, in an epidemiological study, Ohayon et al. (1997) reported VBS with a prevalence of $2 \%$ in the general population. They also reported that night terrors, daytime sleepiness, sleep talking, bruxism, and hypnic jerks were more frequent in subjects with violent or harmful behavior during sleep than the nonviolent subjects, as well as hypnagogic hallucinations,

*Correspondence: myagargunakure.com.tr

Dr. Mehmet Yucel Agargun, Istanbul Medipol University, School of Medicine, Department of Psychiatry, Bağcılar, Istanbul, Turkey

\section{Sleep and Hypnosis Submit your manuscript at www.sleepandhypnosis.org}

the rates of smoking, caffeine and bedtime alcohol intake. There are many neurologic and psychogenic causes of VBS (Pareja et al., 2000). In a clinical and polysomnographic study on 100 consecutive adult patients complaining of sleep-related injury study, Schenck et al. (1989) identified several disorders as being responsible for causing nocturnal violence: sleepwalking, sleep terrors, REM sleep behavior disorder, nocturnal psychogenic dissociative disorders, nocturnal seizures, obstructive sleep apnea, and periodic limb movement disorder.

Nocturnal dissociative disorders, which have been suggested as a type of parasomnia in the literature, may involve violent behavior during sleep. Nocturnal dissociative episodes involve elaborate behaviors that appear as re-enactments of previous abuse situations (e.g., choked or punched by a sibling, beaten or sexually abused by a parent) (Schenck and Mahowald, 2000). Schenck et al. (1989) reported that the associations between nocturnal dissociative episodes and other parasomnias such as sleepwalking and sleep terrors. In addition, self-mutilating behaviors (SMBs) (such as genital 
cutting, self-inflicted burning, and punching through windows) are common in the patients with nocturnal dissociative episodes (Schenck et al., 1989). Psychogenic causes were reported to be more prominent in young population for VBS (Pareja et al., 2000). Thus, it may be suggested that dissociation plays an important role in the underlying mechanisms of both daytime SMBs and violent behaviors during sleep in young population. In the present study, we examined whether there is an association between VBS and dissociative experiences in young adults. We also examined whether VBS is related to daytime SMBs in this population.

\section{METHODS}

Individuals from a representative sample of subjects consisting of undergraduate students in Turkey were included in the study. All students were volunteers and recruited through local announcement for the study. We selected a sample for the study from 382 (253 males and 129 females) undergraduate students. Subjects with organic mental disorders, mental retardation, alcohol and substance abuse, current and past history of psychotic and mood disorders were excluded. Twenty-seven (7\%) of the subjects reported VBS several times throughout their lives. These subjects (23 males and 4 females) were recruited for study group (VBS group). Their mean age was $21.4 \pm 2.1$. Forty-eight subjects ( 27 males and 21 females) who had not ever reported VBS throughout their lives were selected randomly in order to form control group. Their mean age was $20.9 \pm 1.6$. All subjects gave written informed consent prior to their participation for the study. All participants were interviewed in terms of VBS and SMBs using a semi-structured interview questionnaire developed by the authors. The subjects with VBS described their violent behaviors as potentially harmful for themselves or their bed partners. These behaviors included in sexual assault, murder attempt, burning, cutting, and self-mutilating behaviors such as genital cutting, self-inflicted burning, and punching through windows.

The Dissociative Experiences Scale (DES) (7) was used for assessing dissociative experiences. DES is a 28-item self-report scale assessing the frequency of dissociative experiences. Scores range from 0 to 100, and an average score is usually reported. The scale contains a variety of dissociative experiences, many of which are normal experiences. The DES has very good validity and reliability, and good overall psychometric properties. The Turkish version of the scale has reliability and validity as high as its original version (Yargic et al., 1995).

Comparisons with regard to continuous variables were made by means of Student's t-test, and regarding categorical variables, by means of Chi-square test. All significant levels were two-tailed and set at the .05 level, and all the analyses were performed with SPSS 10.0 for Windows.

\section{RESULTS}

The subjects with VBS had significantly higher DES scores than those without VBS $(34.7 \pm 15.3$ vs. $21.3 \pm 13.7$ respectively; $\mathrm{t}=3.86, \mathrm{p}<0.001)$. Twelve $(44.4 \%)$ subjects with VBS and three (6.2\%) subjects without VBS had $S M B s$. The difference between two groups in terms of the presence of SMBs was also significant $\left(\chi^{2}=15.75, p<0.001\right)$.

\section{DISCUSSION}

The present study is the first, to our knowledge, that examines the relationship of dissociative experiences with VBS. The study demonstrates an association among VBS, daytime $S M B s$, and dissociative experiences. The mean DES score of the subjects with VBS were significantly higher than those without VBS. The subjects with VBS were more likely to have daytime SMBs.

VBS is usually caused by neurological conditions in adult or elderly population whereas psychogenic causes are more prominent in young population (Schenck et al., 1989; Ohayon et al., 1997; Pareja et al., 2000). Our sample consist of young subjects, and the present findings suggest that sleep-related violence may be related to dissociative mechanisms, particularly in this population.

Self-mutilation reflects a pathological dissociation. A high incidence of a variety of self-injuries behaviors, including cutting, hitting, burning, stabbing, hair pulling, 
and neurotic excoriations among persons with dissociative disorders (DD) (9). Moreover, it was suggested that "the relative lack of pain may represent a special form of isolation or dissociation" (Butler et al., 1996). Our findings suggest that dissociation may be related to both daytime self-mutilation and violent behaviors during sleep in young adults.

The present study suggests that dissociative experiences are not only related to daytime symptoms in terms of self-mutilation but also may be related to violent behaviors during sleep. It may be concluded that sleeprelated injuries, like parasomnias, promote psychological distress from repeated loss of self-control during sleep. Dissociative mechanisms play an important role in sleeprelated behaviors as well as perception, behavior, memory and identity.

There were some limitations in this study. In the present study, we used only DES to evaluate dissociative experiences, and a structured clinical interview scale was not used to diagnose dissociative disorders. SMBs might be related to diagnosis of DD. Recently, a high incidence of self-injurious behaviors were reported among patients suffering multiple personality disorder, psychogenic amnesia, and DD not otherwise specified (Coons and Milstein, 1990). On the other hand, dysphoria is a hallmark of borderline personality disorder, and this often associated with the initiation of self-mutilating and even suicidal behaviors (Gardner and Cowdry, 1985). Comorbidity with borderline personality disorder or borderline features is high in patients with DD, particularly dissociative identity disorder. Thus, future research is needed to replicate the present findings and to demonstrate the relationship of VBS with comorbidity with borderline personality. Finally, clinical diagnoses were made only based on findings obtained from the clinical interview for VBS, and polysomnographic evaluation was not performed. Future research also should be focused on dissociated states of wakefulness and sleep in terms of violent behaviors and parasomnias using polysomnography.

\section{References}

Bernstein, E.M. \& Putnam, F.W. (1986). Development, reliability, and validity, of a dissociation scale. Journal of Nervous and Mental Disorders 174(12), 727-735.

Butler, L. D., Duran, R. E. F., Jasiukaitis, P., Koopman, C., \& Spiegel, D., (1996). Hypnotizability and traumatic experience: a diathesisstress model of dissociative symptomatology. American Journal of Psychiatry, 153(7), 42-63.

Coons, P. M. \& Milstein, V, 1990. Self-mutilation associated with dissociative disorders. Dissociation, 3(2), 81-87.

Gardner, D. L. \& Cowdry, R. W. (1985). Suicidal and parasuicidal behavior in personality disorder. Psychiatric Clinics of North America, 8(2), 389-403.

Ohayon, M. M., (2000). Violence and sleep. Sleep and Hypnosis, 2(1), 1-7.

Ohayon, M. M., Malijai, C., \& Priets, R. G. (1997). Violent behavior during sleep. Journal of Clinical Psychiatry, 58, 369-376.
Pareja, J. A., Schenck, C. H. \& Mahowald, M. W., (2000). Current perspectives on sleep-related injury, its updated differential diagnosis and its treatment. Sleep and Hypnosis, 2(1), 8-21.

Schenck, C. H. \& Mahowald, M. W. (2000). Parasomnias. Managing bizarre sleep-related behavior disorders. Postgraduate Medicine, 107(3), 145-156.

Schenck, C. H., Milner, D. M., Hurwitz, T. D., Bundlie, S. R., \& Mahowald, M. W. (1989). A polysomnographic and clinical report on sleep-related injury in 100 adult patients. American Journal of Psychiatry, 146(9), 1166-1173.

Schenck, C. H., Milner, D. M., Hurwitz, T. D., \& Bundlie, S. R (1989). Dissociative disorders presenting as somnambulism: polysomnographic, video and clinical documentation (8 cases). Dissociation 2(4), 194-204.

Yargıç, L. I., Tutkun, H., \& Şar, V. (1995). The reliability and validity of the Turkish version of the Dissociative Experiences Scale. Dissociation, 8(1), 10-13. 